\title{
Comment on "Full spin flipping in the presence of full Siberian Snake"
}

\author{
S. R. Mane \\ Convergent Computing Inc., P.O. Box 561, Shoreham, New York 11786, USA
}

(Received 9 February 2009; published 15 September 2009)

\begin{abstract}
Bai and Roser \{Phys. Rev. ST Accel. Beams 11, 091001 (2008) [Phys. Rev. ST Accel. Beams 12, 019901(E) (2009)]\} have published an idea for a design of a spin flipper, consisting of two radial field rf dipoles with correlated phases, to operate with full strength Siberian Snakes, at a spin tune of $\frac{1}{2}$. Some details of their design analysis are oversimplified.
\end{abstract}

DOI: 10.1103/PhysRevSTAB.12.099001

PACS numbers: $29.27 . \mathrm{Hj}$

In a recent paper, Bai and Roser [1] (see also the erratum [2]) have published an idea for a design of a spin flipper, consisting of two radial field rf dipoles with correlated phases, to operate with full strength Siberian Snakes, at a spin tune of $\nu=\frac{1}{2}$. Normally, if a spin flipper is operated at a spin tune of $\frac{1}{2}$, the spin resonance $Q_{\text {osc }}=\nu$ and the "mirror" resonance $1-Q_{\text {osc }}=\nu$ coincide, where $Q_{\text {osc }}$ is the spin flipper tune. The two resonances are crossed in opposite directions, and one does not obtain the condition of an isolated spin resonance as required in the Froissart-Stora formula [3]. The key idea of the design in [1] is that the two rf dipoles are phased so as to cancel the driving term of the mirror resonance. Such a spin flipper can then operate with full strength Siberian Snakes, at a spin tune of $\frac{1}{2}$. However, their design analysis is oversimplified in some respects. The contribution to the resonance strength by the coherent vertical betatron oscillations driven by the $\mathrm{rf}$ dipoles is not completely negligible, as I explain below.

Note that Bai and Roser [1] offer a derivation which indicates that the contributions of the driven vertical betatron oscillations are negligible. They also display a spin tracking simulation to justify that the effects of the driven vertical betatron oscillations are negligible (Fig. 5 of [2]). I ran my own spin tracking simulation, using their values for the spin flipper parameters and tracking, but I obtained different results, as I shall show below.

First, I summarize the principal details of the analysis by Bai and Roser. The spin flipper design is shown in Fig. 3 of [1]. Briefly, it consists of two radial field rf dipoles with a horizontal bend in between (a spin rotation around the vertical axis), and compensating negative horizontal bends outside, so that the horizontal closed orbit excursion is localized within the "flipper system." A sketch of the flipper system is as follows:

$$
\left(-\frac{1}{2} \mathrm{H}\right)-\mathrm{rfd} 1-\mathrm{H}-\mathrm{rfd} 2-\left(-\frac{1}{2} \mathrm{H}\right)
$$

The spin rotation of the central bend is $\phi_{0}$, and those of the outer bends are $-\frac{1}{2} \phi_{0}$ each. The maximum amplitude of the spin rotation by each $\mathrm{rf}$ dipole is $\tilde{\phi}_{\mathrm{osc}}$, i.e.

$$
\tilde{\phi}_{\mathrm{osc}}=\frac{(B L)_{\mathrm{rfd}}}{B \rho}(G \gamma+1) .
$$

The notation is standard: $B \rho$ is the magnetic rigidity, $G=$ $\frac{1}{2}(g-2)$ is the magnetic moment anomaly, and $\gamma$ is the Lorentz factor. Also $(B L)_{\mathrm{rdd}}$ is the peak integrated magnetic field of an rf dipole (the same for both rf dipoles). For later use below, the independent variable is the generalized azimuth $\theta=s / R$, where $s$ is the arclength along the reference orbit and the ring circumference is $2 \pi R$. It is assumed that $\left|\tilde{\phi}_{\text {osc }}\right| \ll 1$, which is a good approximation. The oscillation phases of the rf dipoles are given in [1] as $Q_{\text {osc }} \theta+\chi_{j}$, where $j=1,2$ [see Eq. (12) of [1]; note that $\theta$ is the same for both rf dipoles]. Then the condition to cancel the driving term of the mirror resonance is given as [see Eq. (13) of [1]]

$$
\chi_{1}-\chi_{2}=180^{\circ}+\phi_{0} .
$$

With the above constraint, the resonance strength of the overall spin flipper system is given as [see Eq. (15) of [1]]

$$
\varepsilon=\frac{\tilde{\phi}_{\mathrm{osc}} \sin \phi_{0}}{2 \pi}
$$

Equations (2) and (3) were derived under the assumption that the contribution of the coherent vertical betatron oscillations driven by the rf dipoles is negligible. Bai and Roser [1] presented an argument to justify the above assumption, and they also displayed a spin tracking simulation to demonstrate that the spin flipper would work according to their design calculations. Their simulation used a model relativistic heavy ion collider (RHIC) lattice with full strength Snakes (but without spin rotators) and included the coherent vertical betatron oscillations driven by the rf dipoles. They obtained full spin flip, as shown in Fig. 5 of [2]. In their simulation, the flipper tune was swept from 0.49 to 0.51 in $5 \times 10^{5}$ turns. Additional relevant parameter values were a beam energy of $100 \mathrm{GeV}, \tilde{\phi}_{\text {osc }}=$ $2.28 \mathrm{mrad}, \phi_{0}=30^{\circ}$, and $\chi_{1}-\chi_{2}=210^{\circ}$.

I ran my own spin tracking simulation, using the spin flipper design in [1], with the above parameter values. I should point out that the model RHIC lattice I used is almost certainly not the same as that employed by Bai 
and Roser [1,2]. I contacted the authors to obtain a copy of the same RHIC lattice used in their simulation, but I did not receive a reply. However, the analysis in [1] indicates that the contribution of the driven betatron oscillations to the spin flip should be negligible for basically any RHIC lattice that would be used in normal operations. In the model RHIC lattice I employed, the fractional betatron tunes were $\left(\nu_{x}, \nu_{z}\right)=(0.706,0.715)$ and the beta functions were approximately $40 \mathrm{~cm}$ (in both planes) at both the STAR and PHENIX interaction points. I used a zero emittance beam; the only orbital motion was due to the driven oscillations. I ramped the $\mathrm{rf}$ dipole amplitudes linearly from zero to maximum in 6000 turns at a fixed flipper tune $Q_{\text {osc }}=0.49$ and then swept the flipper tune from 0.49 to 0.51 in a tracking of $5 \times 10^{5}$ turns, and I deexcited the rf dipoles back to zero also in 6000 turns. Following Bai and Roser, I employed full strength Snakes but no spin rotators in the spin tracking. The Snakes were modeled as pointlike $\delta$-function perfect $180^{\circ}$ spin rotations. I also treated the rf dipoles as pointlike $\delta$-function elements.

First, I ran a simulation with the above flipper input values for $\tilde{\phi}_{\text {osc }}, \phi_{0}$, and $\chi_{1}-\chi_{2}$, but I disabled the orbit kicks by the rf dipoles and retained only the spin kicks. Hence, there were no driven orbital oscillations. The results of the spin tracking are displayed in Fig. 1. The plot matches the simulation by Bai and Roser (Fig. 5 of [2]), i.e., full spin flip. I have independently verified Eqs. (2) and (3) in the approximation where the orbit kicks are neglected. Next, I included the orbit kicks and tracked again. The results from my spin tracking simulation are shown in Fig. 2. I found that even after $5 \times 10^{5}$ turns, the vertical component of the spin had not yet settled down to a final

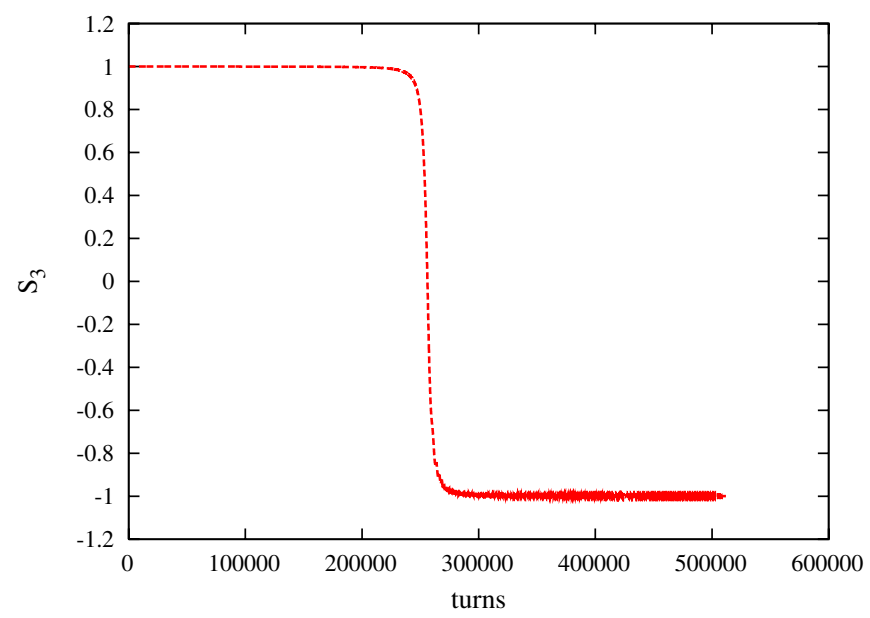

FIG. 1. (Color) Spin tracking simulation of the vertical spin component $S_{3}$ for a model RHIC lattice with $\delta$-function Snakes, no spin rotators, and the spin flipper design in [1]. The orbit kicks by the rf dipoles were disabled and only the spin kicks were retained. The simulation parameters are described in the text. The spin flipper parameter values were $\tilde{\phi}_{\text {osc }}=2.28 \mathrm{mrad}, \phi_{0}=30^{\circ}$, and $\chi_{1}-\chi_{2}=210^{\circ}$.



FIG. 2. (Color) The same spin tracking simulation as in Fig. 1, but now including the orbit kicks by the rf dipoles, i.e., the coherent betatron oscillations driven by the rf dipoles.

asymptotic value, but varied between -0.8 to -1 , in contrast to Fig. 1. Next, I ran the converse simulation and disabled the spin kicks and retained only the orbit kicks by the $\mathrm{rf}$ dipoles. The results of the spin tracking are displayed in Fig. 3. The effect of the orbit kicks is not negligible.

The origin for my tracking was located at the STAR interaction point of RHIC, so $d \beta_{z} / d s=0$, so I calculated the analog of the normalized Courant-Snyder invariant via

$$
\mathcal{W}_{\mathrm{coh}}=\beta \gamma\left[\frac{z_{*}^{2}}{\beta_{z^{*}}}+\beta_{z *}\left(\frac{d z_{*}}{d s}\right)^{2}\right]
$$

The vertical coordinate is $z$ and $\beta_{z}$ is the vertical beta function. (I shall define my coordinate system in more detail later in this comment.) The asterisks indicate values measured at the origin and the factor of $\beta \gamma(=106.6$ at $100 \mathrm{GeV}$ ) is the same as in the definition of the normalized

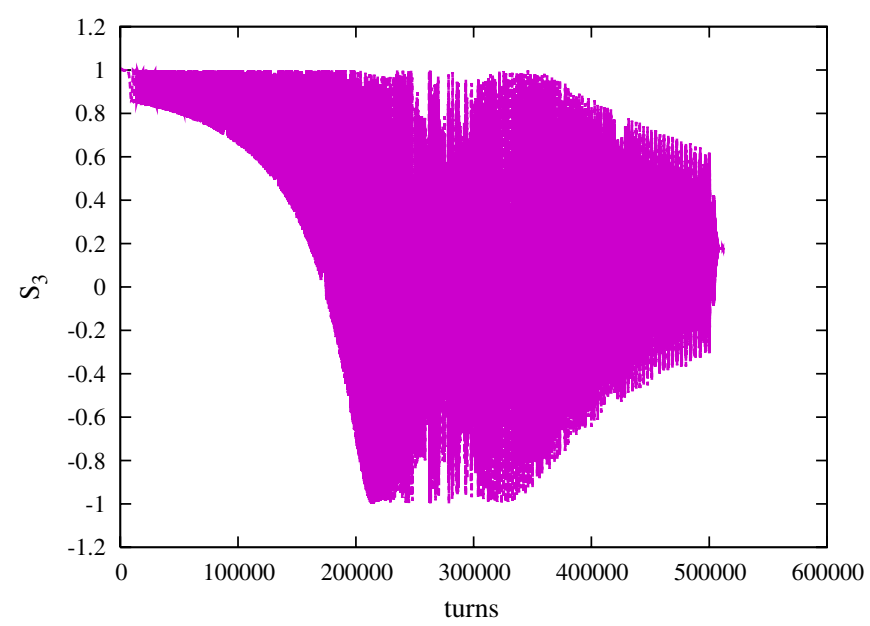

FIG. 3. (Color) The same spin tracking simulation as in Fig. 1, but now the spin kicks by the rf dipoles were disabled and only the orbit kicks were retained. 
emittance. Note, however, that $\mathcal{W}_{\text {coh }}$ is not an invariant of the motion for driven betatron oscillations. The maximum value of $\mathcal{W}_{\text {coh }}$ that I obtained in my tracking was $\max \left(\mathcal{W}_{\text {coh }}\right) \simeq 0.128 \pi \mathrm{mmmrad}$. This is indeed much smaller than the rms emittance of $2.5 \pi \mathrm{mm}$ mrad of the intrinsic vertical betatron oscillations that is mentioned in [1]. Hence, I can confirm the statement in [1] that the amplitude of the driven oscillations is small, but that does not mean the contribution to the spin flip resonance strength is negligible.

I ran some other benchmark tests. A significant feature of the flipper design in [1] is that the cancellation of the mirror resonance is self-contained within the flipper system, and does not depend on the details of the rest of the ring. This property is also independent of the details of the RHIC lattice. See Eq. (17) of [1] for the one-turn spin matrix: the only property required from the rest of the ring is that it should yield an overall spin rotation of $180^{\circ}$ around the vertical axis. I therefore ran the exact same spin tracking simulation as in Fig. 2 again (i.e. including both orbit and spin kicks by the rf dipoles), but with spin rotators configured to deliver longitudinal polarization at the PHENIX interaction point (the polarization at the origin, i.e., STAR, was vertical). I modeled the spin rotators as pointlike $\delta$-function elements with no effect on the orbital motion. The results of the spin tracking are displayed in Fig. 4. The orbital motion is exactly the same as in Fig. 2, because the Snakes and spin rotators do not affect the orbital motion in my simulation. However, the behavior of the spin is very different from Fig. 2, and is not a successful spin flip.

Another test that the flipper design is self-contained is that the spin flipper should also work for a flat ring, i.e., without Snakes and spin rotators, provided only that the fractional spin tune is $\frac{1}{2}$. I therefore ran the same spin

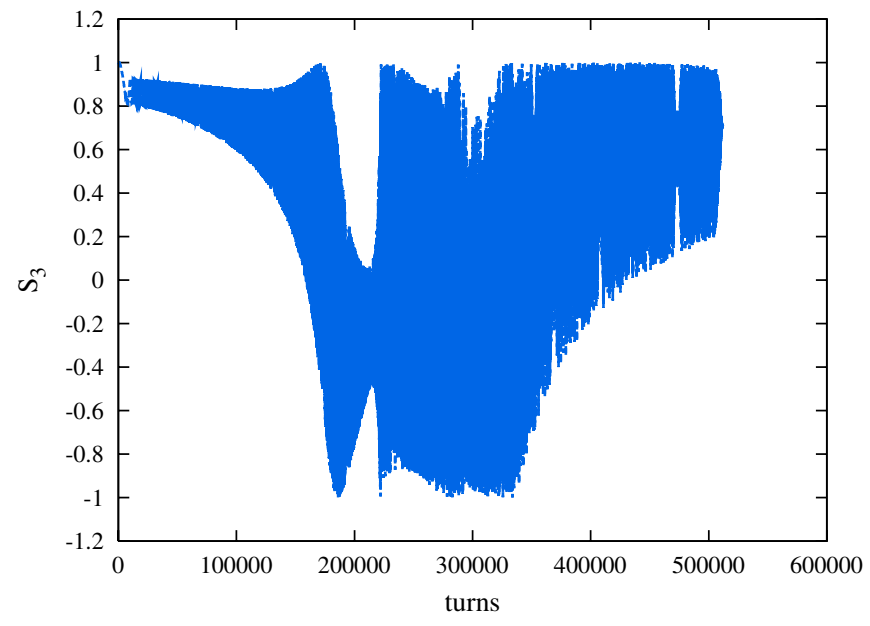

FIG. 4. (Color) The same spin tracking scenario as in Fig. 2, but including spin rotators configured for longitudinal polarization at the PHENIX interaction point of RHIC. The polarization at the STAR interaction point was vertical. tracking as in Fig. 2, but for a flat RHIC lattice without Snakes and spin rotators. Since $G \gamma=191.08$ at $100 \mathrm{GeV}$, I increased the beam energy slightly and ran the simulation at $G \gamma=191.5$, i.e., a fractional spin tune of $\frac{1}{2}$. I again obtained $\max \left(\mathcal{W}_{\text {coh }}\right) \simeq 0.128 \pi \mathrm{mm}$ mrad, because the orbital motion is not affected by the presence or absence of Snakes and spin rotators, and the change to the beam energy was small. The results of the spin tracking are displayed in Fig. 5. It is not a successful spin flip.

I now present my theoretical findings. The description in [1] of the calculations for the driven vertical betatron oscillations is very compressed, so I shall explain in my own words. First I need some additional notation. I have already denoted the spin tune by $\nu$, and I define the symbol $\nu_{0}=G \gamma$, and a prime denotes differentiation with respect to $\theta$, i.e. $f^{\prime}=d f / d \theta$ for any function $f$. Unfortunately, there is a variety of coordinate systems in common use by workers in the field, but most of my theoretical formulas are taken from Courant and Ruth [4] and Ptitsyn [5], so I follow them and employ the coordinate system $(\hat{\boldsymbol{x}}, \hat{\boldsymbol{y}}, \hat{z})$, where $x$ is radial, $y$ is longitudinal, and $z$ is vertical. (Note that Bai and Roser [1] use $y$ to denote the vertical coordinate.) The positive sense of circulation is counterclockwise around the ring.

For the orbital motion, to leading order the Snakes and spin rotators in RHIC are "optically transparent" and are equivalent to drift spaces of the same length as the space they occupy. In particular, they do not generate any transverse betatron coupling. Then Hill's equation for the forced vertical oscillations $z_{\text {coh }}$ from a single rf dipole at $\theta_{0}$ is (I drop subscripts on $B L$ and $\chi$ )

$$
z_{\mathrm{coh}}^{\prime \prime}+g_{z} z_{\mathrm{coh}}=R \frac{B L}{B \rho} \cos \left(Q_{\mathrm{osc}} \theta+\chi\right) \delta_{p}\left(\theta-\theta_{0}\right) .
$$

Here $g_{z}$ is the vertical focusing function and the periodic $\delta$ function is

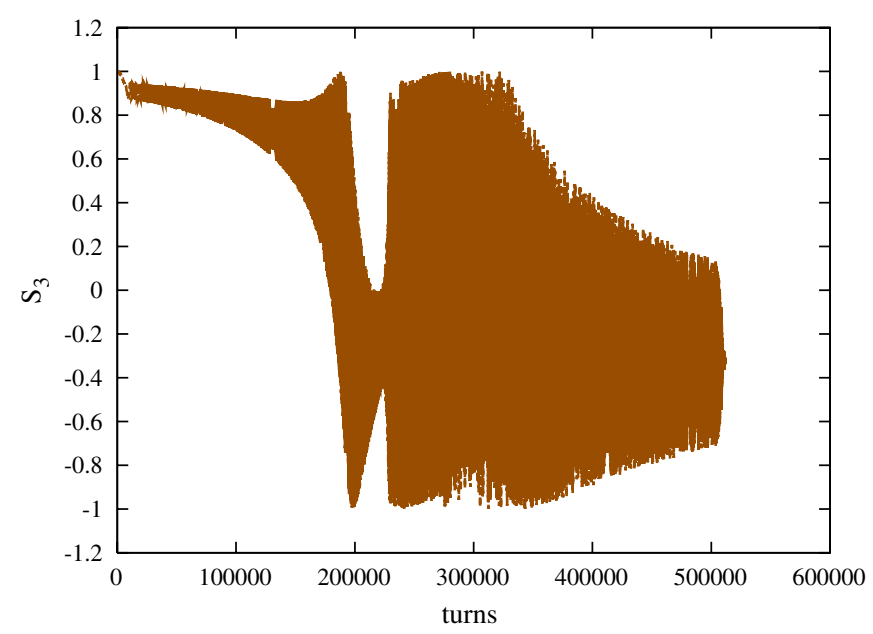

FIG. 5. (Color) The same spin tracking simulation as in Fig. 2, but for a flat RHIC lattice without Snakes and spin rotators, at a spin tune of $G \gamma=191.5$, i.e., a fractional spin tune of $\frac{1}{2}$. 


$$
\delta_{p}\left(\theta-\theta_{0}\right)=\sum_{j=-\infty}^{\infty} \delta\left(\theta-\theta_{0}-2 j \pi\right) .
$$

The solution for the driven betatron oscillation is

$$
\begin{aligned}
z_{\mathrm{coh}}= & \frac{\nu_{z} \beta_{z 0}^{1 / 2} \beta_{z}^{1 / 2}}{2 \pi} \frac{B L}{B \rho} \\
& \times \sum_{k=-\infty}^{\infty} \frac{\cos \left[\left(k+Q_{\mathrm{osc}}\right) \psi+\chi-k \psi_{0}\right]}{\nu_{z}^{2}-\left(k+Q_{\mathrm{osc}}\right)^{2}} .
\end{aligned}
$$

Here $\beta_{z 0}$ is the value of the vertical beta function at $\theta_{0}, \nu_{z}$ is the vertical betatron tune, $\psi=\phi_{z} / \nu_{z}$, and the betatron phase is

$$
\phi_{z}=\int_{0}^{\theta} \frac{R}{\beta_{z}} d \tilde{\theta}
$$

Bai and Roser [1] write the following expression for the amplitude of the driven betatron oscillation [see Eq. (20) of [1], with obvious changes of notation]:

$$
z_{\mathrm{amp}}=\frac{\nu_{z} \beta_{z 0}^{1 / 2} \beta_{z}^{1 / 2}}{2 \pi} \frac{B L}{B \rho} \sum_{k=-\infty}^{\infty} \frac{1}{\left|\nu_{z}^{2}-\left(k+Q_{\mathrm{osc}}\right)^{2}\right|} .
$$

Essentially, each term in the sum in Eq. (7) is substituted by its maximum amplitude, to obtain Eq. (9). It is then stated in [1] that the amplitude of the driven betatron oscillation in Eq. (9) is dominated by the harmonic nearest to the betatron tune [see Eq. (21) of [1]].

To proceed further, I show the integral for the spin resonance strength. For a planar uncoupled ring without Snakes or spin rotators, the resonance strength is given by

$$
\varepsilon=-\frac{i}{2 \pi} \oint\left[\left(\nu_{0}^{2}+G\right) \frac{z_{\text {res }}^{\prime}}{\rho}+(1+G)\left(\frac{1}{\rho}\right)^{\prime} z_{\text {res }}\right] e^{i \nu_{0} \Theta} d \theta .
$$

Here $z_{\text {res }}$ is the resonant part of the vertical orbital motion, $\rho$ is the local bend radius in the horizontal plane, and

$$
\Theta=\int_{0}^{\theta} \frac{R}{\rho(\tilde{\theta})} d \tilde{\theta} .
$$

The integral in Eq. (10) coincides (up to an integration by parts and differences of notation) with that given by Courant and Ruth [4]. For an imperfection resonance, $z_{\text {res }}$ denotes the imperfect closed orbit, while for an intrinsic resonance, $z_{\text {res }}$ denotes a free vertical betatron oscillation. Equation (10) must be generalized in two ways, to describe the resonance strength for a spin flipper (consisting of rf dipoles) in a ring with Snakes and spin rotators: (i) The value of $z_{\text {res }}$ must be the coherent betatron oscillation driven by the spin flipper. (ii) The phase factor $e^{i \nu_{0} \Theta}$ must be generalized to a function which describes the spin precession in a ring with Snakes and spin rotators. For now, however, I continue with the analysis in [1]. Bai and Roser calculate the resonance strength for the intrinsic oscillations (using an rms emittance of $2.5 \pi \mathrm{mm} \mathrm{mrad}$ ) for a flat ring without Snakes and spin rotators, obviously using Eq. (10) or an equivalent formula, and obtain a value of about 0.001 . Next, they compare the amplitude of the driven oscillations (using an integrated field of 40 Gauss-m for each rf dipole, and $100 \mathrm{GeV}$ beam energy) and find it to be about one-tenth of the rms beam size, and this ratio of amplitudes is used to estimate the resonance strength due to the driven oscillations to be approximately $3 \times 10^{-5}$. It is stated in [1] that "With snakes, the effect of driven betatron oscillation on spin motion is further reduced." (This is not true, as I shall explain below.) From [1], the effective maximum spin rotation of the two rf dipoles is about 0.0012 . Hence, it is deduced in [1] that the contribution of the driven oscillations to the spin flip resonance strength is negligible. However, my tracking simulation even for a flat ring without Snakes (see Fig. 5) does not support the claim that the driven oscillations are negligible.

I now present my own analysis of the spin flip resonance strength. First, the values I obtained for $\max \left(\mathcal{W}_{\text {coh }}\right)$ in my tracking simulations are consistent with the statement in [1] that the amplitude of the driven oscillations is about one-tenth of the rms beam size, for an rms emittance of $2.5 \pi \mathrm{mm}$ mrad. Hence, I can confirm this aspect of the analysis in [1]. Second, an effective rotation of 0.0012 means $\varepsilon=0.0012 /(2 \pi) \simeq 1.9 \times 10^{-4}$ in Eq. (3). Hence, the stated contribution of $3 \times 10^{-5}$ from the driven oscillations is not a priori negligible. (No calculation is given as to how much the Snakes reduce the contribution of the driven oscillations, and in fact I shall point out below that they do not reduce it at all.) However, third and most importantly, there is no need to perform any approximate calculations using a formula such as Eq. (10) for a flat ring without Snakes and spin rotators, followed by estimates of the effects of Snakes. The relevant resonance strength formula, for rings with Snakes and spin rotators, is known. I display it below. The notation below mainly follows [5]. The spin precession equation of motion in the externally prescribed electric and magnetic fields of the accelerator is known as the Thomas-Bargmann-Michel-Telegdi equation $[6,7]$. Using $\theta$ as the independent variable, the equation takes the form $d s / d \theta=\boldsymbol{W} \times \boldsymbol{s}$, where $\boldsymbol{s}$ is the classical spin vector. I subdivide the spin precession vector into two parts $\boldsymbol{W}=\boldsymbol{W}_{0}+\boldsymbol{w}$, where $\boldsymbol{W}_{0}$ is the spin precession vector on the ideal (reference) orbit, and $\boldsymbol{w}$ describes the off-axis motion. The spin motion due to $\boldsymbol{w}$ is treated as a perturbation (this includes spin flippers). The unperturbed spin precession equation is $d \boldsymbol{s} / d \theta=\boldsymbol{W}_{0} \times \boldsymbol{s}$. It has three linearly independent solutions. The solution sets contain an infinity of possible right-handed orthonormal triads. One possibility is to choose the following set of spin basis vectors. One of the solutions, denoted by the unit vector $\boldsymbol{n}_{0}$, is periodic after one turn round the ring: $\boldsymbol{n}_{0}(\theta+2 \pi)=$ $\boldsymbol{n}_{0}(\theta)$. Denote the two other linearly independent solutions on the reference orbit by the unit vectors $\boldsymbol{\eta}_{1}$ and $\boldsymbol{\eta}_{2}$, respectively, and let $\left\{\boldsymbol{n}_{0}, \boldsymbol{\eta}_{1}, \boldsymbol{\eta}_{2}\right\}$ be a right-handed ortho- 
normal triad. The vectors $\boldsymbol{\eta}_{1}$ and $\boldsymbol{\eta}_{2}$ are not periodic after one turn. Define $\boldsymbol{\eta} \equiv \boldsymbol{\eta}_{1}-i \boldsymbol{\eta}_{2}$, then $\boldsymbol{\eta}(\theta+2 \pi)=$ $e^{i 2 \pi \nu} \boldsymbol{\eta}(\theta)$. The normalization is $\left|\boldsymbol{n}_{0}\right|^{2}=1$ and $|\boldsymbol{\eta}|^{2}=2$. In a flat ring without Snakes, $\boldsymbol{n}_{0}=\hat{z}, \boldsymbol{\eta}=(\hat{\boldsymbol{x}}-i \hat{\boldsymbol{y}}) e^{i \nu_{0} \Theta}$, and $\nu=\nu_{0}$. Then $\eta_{y}=-i e^{i \nu_{0} \Theta}$ is the phase factor in Eq. (10). For RHIC, $\nu=\frac{1}{2}$ and $\boldsymbol{n}_{0}$ and $\boldsymbol{\eta}$ will depend on the Snake and spin rotator configurations. To first order, the spin resonance strength is given by the following one-turn integral:

$$
\varepsilon=\frac{1}{2 \pi} \oint \boldsymbol{w}_{\mathrm{res}} \cdot\left(\boldsymbol{\eta}_{1}-i \boldsymbol{\eta}_{2}\right) d \theta=\frac{1}{2 \pi} \oint \boldsymbol{w}_{\mathrm{res}} \cdot \boldsymbol{\eta} d \theta .
$$

Here $\boldsymbol{w}_{\text {res }}$ contains only resonant terms, and must be evaluated at the center of the spin resonance. (Then the integrand is periodic around the ring.) The calculation/ estimation of resonance strengths by Bai and Roser [see Eq. (10)] is based on models without Snakes, i.e. $\boldsymbol{n}_{0}$ is vertical everywhere. However, for a ring with a pair of Snakes, such as RHIC, $\boldsymbol{n}_{0}=\hat{z}$ in one arc and reverses direction $\boldsymbol{n}_{0}=-\hat{\boldsymbol{z}}$ in the other arc. The behavior of $\boldsymbol{n}_{0}$ is even more complicated near the interaction points, when spin rotators are switched on. The vectors $\boldsymbol{\eta}_{1}$ and $\boldsymbol{\eta}_{2}$ also vary in complicated ways when Snakes and spin rotators are present. The integrand in Eq. (12), and hence the resonance strength $\varepsilon$, thus depends on the lattice optics, and, in particular, on the configuration of the spin rotators. It is not a priori valid to employ an approximation where $\boldsymbol{n}_{0}=\hat{z}$ always, and the configuration of the spin rotators is ignored. The integral for the spin resonance strength, for a ring with Snakes and spin rotators (but without transverse betatron coupling), is

$$
\varepsilon=\frac{1}{2 \pi} \oint\left[\left(\nu_{0}^{2}+G\right) \frac{z_{\text {res }}^{\prime}}{\rho}+(1+G)\left(\frac{1}{\rho}\right)^{\prime} z_{\text {res }}\right] \eta_{y} d \theta .
$$

This formula generalizes Eq. (10). The formalism in [5] can in addition treat transverse coupling. See also a formula using so-called "spin response" functions below. For a spin flipper consisting of multiple rf dipoles, the value of $z_{\text {res }}$ is the sum of the coherent betatron oscillations driven by all the rf dipoles. Note that Eq. (13) includes the contributions of both the direct spin kicks by the rf dipoles and the indirect contribution of the spin-orbit coupling due to the driven betatron oscillations around the rest of the ring. For an rf dipole located at $\theta_{0}$, the relevant harmonics in the sum in Eq. (7) are those which contribute the most to the integral in Eq. (13). It does not matter if those harmonics are not the closest to the betatron tune, or if they do not have the largest magnitudes in the sum in Eq. (9).

These ideas can be easily checked using a smooth focusing model. I display the solution for a smooth focusing model with a pair of diametrically opposed orthogonal Snakes at $\theta=0$ and $\pi$, with axes at $\pm 45^{\circ}$ to the beam direction, respectively (like RHIC). Let the origin be just after the location of the first Snake. I treat the Snakes as pointlike $\delta$-function objects. Then (outside of the Snakes) $\boldsymbol{n}_{0}= \pm \hat{z}$ respectively in the two arcs, and

$$
\boldsymbol{\eta}= \begin{cases}(\hat{\boldsymbol{x}}-i \hat{\boldsymbol{y}}) e^{i \nu_{0} \Theta} & (0<\theta<\pi) \\ (i \hat{\boldsymbol{x}}-\hat{\boldsymbol{y}}) e^{i \nu_{0}(2 \pi-\Theta)} & (\pi<\theta<2 \pi) .\end{cases}
$$

For a smooth focusing model $\Theta=\psi=\theta, \quad \rho=R$, $(1 / \rho)^{\prime}=0$, and $\beta_{z}=R / \nu_{z}$. The resonant tune is $Q_{\text {osc }}=$ $\frac{1}{2}$. The driven oscillation for one rf dipole located at $\theta_{0}$ can be subdivided into $z_{\mathrm{coh}}=z_{+}+z_{-}$(driving terms for resonance and mirror), where $z_{-}=z_{+}^{*}$ and

$$
z_{+}=\frac{R}{4 \pi} \frac{B L}{B \rho} e^{-i\left[(1 / 2) \theta_{0}+\chi\right]} \sum_{k=-\infty}^{\infty} \frac{e^{-i[k+(1 / 2)]\left(\theta-\theta_{0}\right)}}{\nu_{z}^{2}-\left(k+\frac{1}{2}\right)^{2}} .
$$

For brevity define $\kappa=k+\frac{1}{2}= \pm \frac{1}{2}, \pm \frac{3}{2}, \ldots$ below. The resonance strength due to $z_{+}$can be expressed in the form

$$
\varepsilon=\frac{1}{4 \pi} \frac{B L}{B \rho} e^{-i\left[(1 / 2) \theta_{0}+\chi\right]} \mathcal{F}\left(\theta_{0}\right) .
$$

Evaluating the integral in Eq. (13), the solution for $\mathcal{F}$ is

$$
\begin{aligned}
\mathcal{F}\left(\theta_{0}\right)= & \frac{i}{2 \pi}\left(\nu_{0}^{2}+G\right) \sum_{\kappa= \pm(1 / 2), \pm(3 / 2), \ldots} \frac{\kappa e^{i \kappa \theta_{0}}}{\left(\nu_{z}^{2}-\kappa^{2}\right)\left(\nu_{0}^{2}-\kappa^{2}\right)} \\
& \times\left\{\nu_{0}+\kappa+i\left(\nu_{0}-\kappa\right)\right. \\
& \left.-\left[\nu_{0}+\kappa-i\left(\nu_{0}-\kappa\right)\right] e^{i\left(\nu_{0}-\kappa\right) \pi}\right\} .
\end{aligned}
$$

The above expression for $\mathcal{F}$ equals that for the "spin response" function $F_{3}$ which I calculated for the same model in [8]. The full value of the resonance strength is obtained by summing the contributions from both rf dipoles, but provided the fractional part of $\nu_{z}$ is not very close to $\frac{1}{2}$, there is no particular term of the sum indexed by $\kappa$ which dominates the above sum. I discuss this in more detail as follows. First, when $\nu_{0} \equiv G \gamma \simeq n+\frac{1}{2}$ (for some integer $n$ ) so that there is a $\kappa_{0}$ with $\left|\nu_{0}-\kappa_{0}\right| \ll 1$, the relevant term stays bounded and does not dominate over the rest. Second, for arbitrary $\nu_{0}$ and the fractional part of $\nu_{z}$ is not close to $\frac{1}{2}$, the terms are asymptotically bounded from above by $O(1) / \kappa^{2}$ such that the sum converges absolutely. Third, when $\nu_{z}$ approaches $n+\frac{1}{2}$, so that there is a $\kappa_{z}$ with $\left|\nu_{z}-\kappa_{z}\right| \ll 1$, then there is one dominant (in fact diverging) term. However, this occurs only if the fractional vertical betatron tune is close to $\frac{1}{2}$, in which case the rf dipoles do indeed drive the beam at the vertical betatron frequency. However, it is clearly stated by Bai and Roser [1] that the operating point of the ring is such that the fractional vertical betatron tune is not close to $\frac{1}{2}$. See also my discussion below in connection with Fig. 6, about the crossing of spin resonances when accelerating the beam energy, or using a spin flipper. Also, comparing to Eqs. (1) and (3), there is no reason why the value of $\mathcal{F}$ should be smaller than $O(G \gamma+1)$, and indeed it can be larger.

For nonsmooth models, there exists an explicit integral for the spin flip resonance strength in terms of lattice optical functions. First I define the dimensionless scaled 


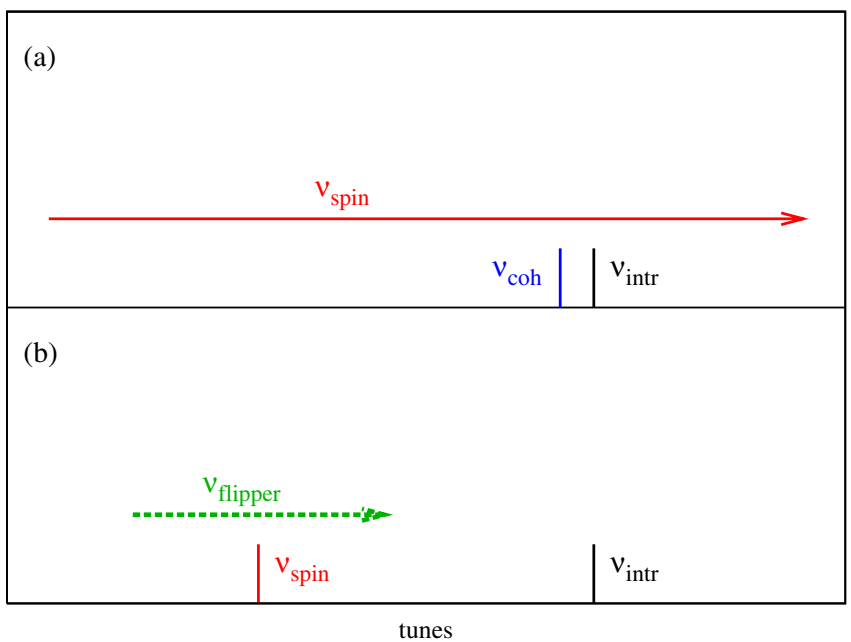

FIG. 6. (Color) Schematic description of two scenarios of tune sweeps. In (a), the spin tune is swept across the tune of the intrinsic oscillations and also the driven oscillations ( $\mathrm{rf}$ dipole run at a fixed tune), where the coherent and intrinsic tunes are almost equal. This scenario corresponds to the crossing of an intrinsic resonance using an $\mathrm{rf}$ dipole to adiabatically induce a large amplitude coherent betatron oscillation, to achieve adiabatic spin flip without emittance blowup. Scenario (b) describes a spin flipper operation, where the spin tune is fixed. The spin flipper tune (coherent oscillation tune) is swept across the spin tune, but the intrinsic oscillation tune does not cross the spin tune.

vertical magnetic field in the ring dipoles $K_{z}=R / \rho$. I also define the dimensionless quantities (with an obvious notation for $j=1,2$ )

$$
\Delta K_{x j}=\frac{1}{2} \frac{(B L)_{j}}{B \rho} e^{-i\left(Q_{\mathrm{osc}} \theta_{1}+\chi_{j}\right)} .
$$

Note that, following Bai and Roser [1], I employ $\theta_{1}$ for both rf dipoles, so my definition of $\chi_{1.2}$ is the same as theirs. The resonance strength is [5]

$$
\varepsilon=\frac{1}{2 \pi} \sum_{j=1,2}\left[\Delta K_{x j} F_{3}\left(\theta_{j}\right)\right]_{\nu=Q_{\mathrm{osc}}=(1 / 2)}
$$

Here $F_{3}$ is a so-called "spin response function" [5] and is a function of the lattice optics. For uncoupled orbital motion, as at RHIC, the expression for $F_{3}$ is given in terms of lattice optical functions via [5]

$$
\begin{aligned}
F_{3}\left(\theta_{j}\right)= & \frac{i}{2}\left\{\frac { f _ { z } ( \theta _ { j } ) } { e ^ { i 2 \pi ( \nu - \nu _ { z } ) } - 1 } \int _ { \theta _ { j } } ^ { \theta _ { j } + 2 \pi } \left[f_{z}^{* \prime}\left(\nu_{0}^{2}+G\right) K_{z} \eta_{y}\right.\right. \\
& \left.+f_{z}^{*}(1+G) K_{z}^{\prime} \eta_{y}\right] d \theta \\
& -\frac{f_{z}^{*}\left(\theta_{j}\right)}{e^{i 2 \pi\left(\nu+\nu_{z}\right)}-1} \int_{\theta_{j}}^{\theta_{j}+2 \pi}\left[f_{z}^{\prime}\left(\nu_{0}^{2}+G\right) K_{z} \eta_{y}\right. \\
& \left.\left.+f_{z}(1+G) K_{z}^{\prime} \eta_{y}\right] d \theta\right\} .
\end{aligned}
$$

Here $f_{z}=\sqrt{\beta_{z} / R} e^{i \phi_{z}}$. We set $\nu=\frac{1}{2}$ in the resonance denominators in Eq. (20). The value of $F_{3}$ depends on the details of the RHIC lattice and also on the configuration of the spin rotators. The flipper design in [1] does not depend on the configuration of the spin rotators. I have pointed out this fact above, see Fig. 4. See [9] for further details, including numerical calculations of $F_{3}$ for a model RHIC lattice (it is found that the driven oscillations dominate the contribution to the value of $\left|F_{3}\right|$ ).

I calculated the value of $F_{3}$ analytically [8] for smooth focusing models with one Snake and a pair of pointlike $\delta$-function diametrically opposed orthogonal Snakes. I showed for the two-Snake model that the amplitude $\left|F_{3}\right|$ can be larger in a ring with Snakes than in the same model without Snakes. Also in [9] the value of $F_{3}$ was calculated for a RHIC lattice with and without Snakes and it was shown that the value of $\left|F_{3}\right|$ in RHIC is not reduced by the presence of Snakes. This is in disagreement with the statement in [1] quoted above: "With snakes, the effect of driven betatron oscillation on spin motion is further reduced."

Note that for polarized beam acceleration to high energy, the use of Siberian Snakes makes the spin tune independent of the beam energy, and it is for this reason the spin precession does not resonate with the intrinsic oscillations or closed orbit imperfections. The use of Siberian Snakes therefore greatly reduces (or eliminates) depolarization during acceleration to high energy. However, it is different for driven oscillations which are deliberately resonant with the spin precession. The use of Snakes does not necessarily reduce the effects of resonant orbital oscillations on the spin precession.

The analysis in [1] seems to be based on an analogy with the use of an $\mathrm{rf}$ dipole to adiabatically induce a large amplitude coherent betatron oscillation, to (successfully) achieve adiabatic spin flip without emittance blowup, while sweeping the spin tune in the alternating-gradient synchrotron (AGS) across an intrinsic resonance [10]. The matter is best explained using a diagram, as in Fig. 6. In the scenario described in Fig. 6(a), an rf dipole is run at a fixed tune $\nu_{\text {osc }}$ which is deliberately very close to the intrinsic betatron tune $\nu_{\text {intr. }}$. The rf dipole drives a large amplitude coherent vertical betatron oscillation. The spin tune is swept across the tunes of both the coherent and the intrinsic betatron oscillations. Hence, the spin precession resonates with both the coherent and the intrinsic betatron oscillations. This was the scenario in [10]. It is valid in this case to compare the resonance strengths due to the coherent and intrinsic betatron oscillations by comparing the amplitudes of the coherent and the intrinsic oscillations. Furthermore, because the tune of the coherent oscillations was almost equal to the tune of the intrinsic oscillations, the amplitude of the coherent oscillations really was dominated by just a single term in the sum in Eq. (9): there is one term in the sum whose denominator is much smaller than that of any 
other term. However, the scenario is different for a spin flipper operation, even though the flipper is also an rf dipole. The scenario is described in Fig. 6(b). Now the spin tune is held fixed, and it does not cross the tune of the intrinsic betatron oscillations. Instead the tune of the $r f$ dipole (the spin flipper) is swept across the (fixed) spin tune. The essential point to note in this scenario is that the spin precession is resonant with only the coherent betatron oscillations and not the intrinsic betatron oscillations. The intrinsic vertical betatron oscillations constitute a nonresonant background term. This is the key distinction between the two scenarios in Fig. 6. It then does not matter if the amplitude of the driven betatron oscillations is smaller than that of the rms intrinsic vertical betatron oscillations: the coherent oscillations drive a spin flip and the intrinsic oscillations do not.

It is stated in [1] that "For accelerators like RHIC where the spin precession tune is half integer, the rf dipole tune then is required to be swept through half integer for the spin flipping. However, since in general the betatron tune is kept away from half integer for stability reasons, the amplitude of the driven oscillation is insignificant and this effect, as well as the resulting contribution to the rf dipole spin resonance, is negligible." While it is true that the amplitude of the driven oscillations is "small," this does not imply "insignificant." The driven oscillations are resonant with the spin precession, and their contribution to the spin flip resonance strength can be calculated quantitatively via Eqs. (19) and (20).

I close with a brief remark on the idea in [1] to measure the spin tune by running the rf dipoles at a fixed tune close to the spin tune. I ran a spin tracking simulation using Snakes and no spin rotators, with $Q_{\mathrm{osc}}=0.498$ so $\delta=$ $0.5-Q_{\text {osc }}=0.002$ and the same flipper parameters as in Fig. 2 , so $\varepsilon=0.00114 /(2 \pi) \simeq 1.8 \times 10^{-4}$. The solution for the vertical and horizontal spin components is given in Eqs. (18) and (19) of [1] and for my inputs one expects the amplitudes of the vertical and horizontal spin components to be

$$
\begin{gathered}
S_{\text {vert }}=\frac{\delta}{\sqrt{\varepsilon^{2}+\delta^{2}}} \simeq 0.996, \\
S_{\text {horz }}=\frac{\varepsilon}{\sqrt{\varepsilon^{2}+\delta^{2}}} \simeq 0.09 .
\end{gathered}
$$

It is stated in [1] that the value of the vertical spin component will be constant turn by turn. The results from my spin tracking simulation are shown in Fig. 7. The rf dipoles were ramped linearly from zero to maximum amplitude in 6000 turns, run at flattop for 12000 turns, and ramped linearly back to zero in 6000 turns. The plotted spin components are $S_{1}=\boldsymbol{s} \cdot \boldsymbol{\eta}_{1}, S_{2}=\boldsymbol{s} \cdot \boldsymbol{\eta}_{2}$, and $S_{3}=\boldsymbol{s} \cdot \boldsymbol{n}_{0}$. At the origin $\boldsymbol{n}_{0}=\hat{z}$ every turn, while $\boldsymbol{\eta}_{1}=(-1)^{k} \hat{\boldsymbol{x}}$ and $\boldsymbol{\eta}_{2}=(-1)^{k} \hat{\boldsymbol{y}}$ on the $k$ th turn. This yields smoother plots for $S_{1}$ and $S_{2}$, which otherwise reverse sign every turn. Contrary to the

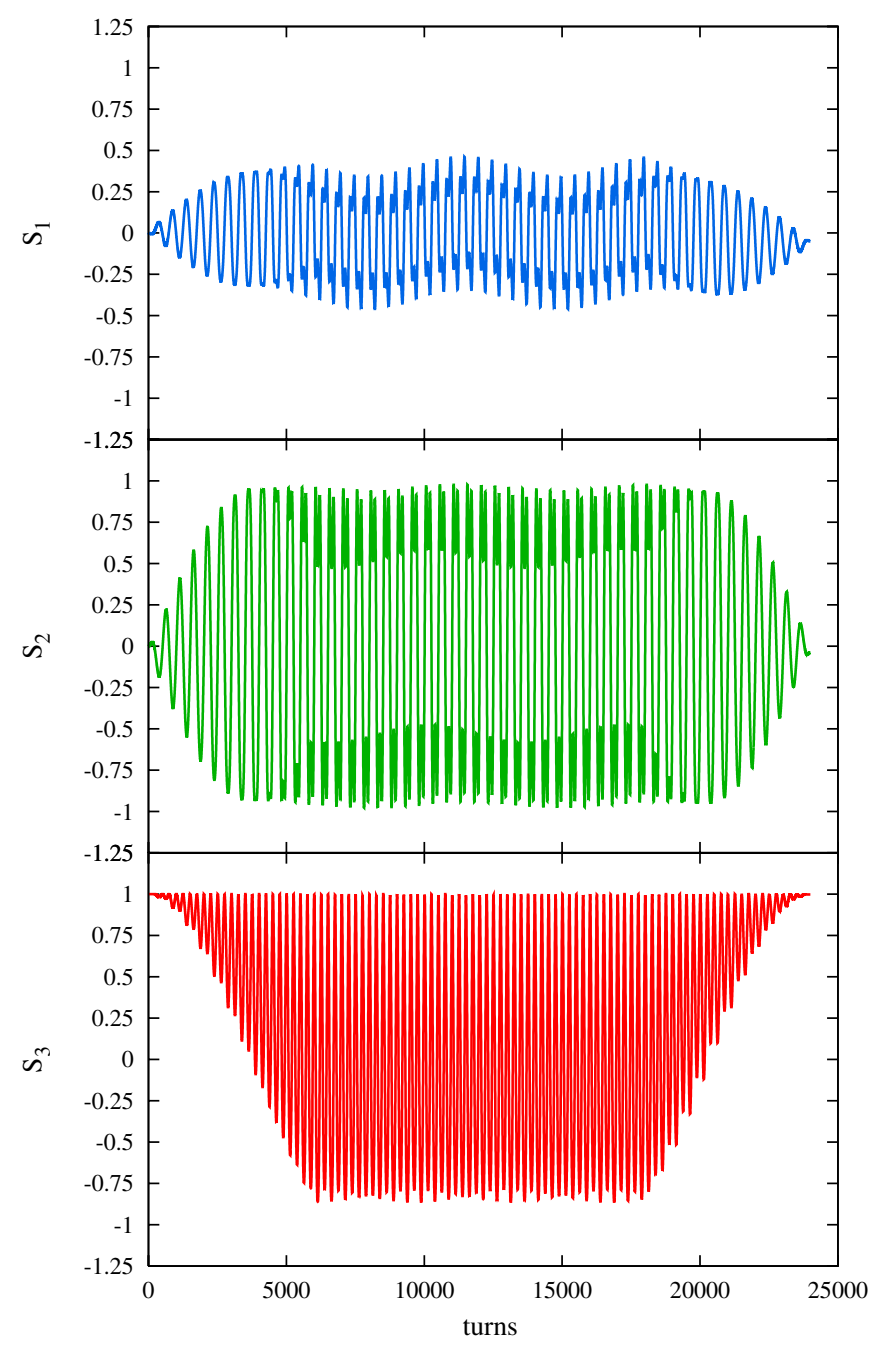

FIG. 7. (Color) Graphs of the spin components $S_{1}=\boldsymbol{s} \cdot \boldsymbol{\eta}_{1}$, $S_{2}=\boldsymbol{s} \cdot \boldsymbol{\eta}_{2}$, and $S_{3}=\boldsymbol{s} \cdot \boldsymbol{n}_{0}$, when the spin flipper is run at a fixed tune of $Q_{\text {osc }}=0.498$. The flipper parameters are the same as in Fig. 2. The rf dipoles were ramped linearly from zero to maximum amplitude in 6000 turns, run at flattop for 12000 turns, and ramped linearly back to zero in 6000 turns.

claim in [1], the value of the vertical spin component $S_{3}$ is not constant turn by turn at flattop, but goes almost from +1 to -0.8 . Also, the maximum amplitudes of the spin components $S_{1}$ and $S_{2}$ are unequal. Basically, the mirror resonance has not been canceled by the condition in Eq. (2). In general, the spin tracking results will also depend on the configuration of the spin rotators. I have verified that one does approximately obtain the solution in Eq. (21) if one runs a simulation where the orbit kicks from the rf dipoles are disabled. The results in Fig. 7 are due to the effects of the driven betatron oscillations.

[1] M. Bai and T. Roser, Phys. Rev. ST Accel. Beams 11, 091001 (2008). 
[2] M. Bai and T. Roser, Phys. Rev. ST Accel. Beams 12, 019901(E) (2009).

[3] M. Froissart and R. Stora, Nucl. Instrum. Methods Phys. Res., Sect. A 7, 297 (1960) (in French).

[4] E.D. Courant and R.D. Ruth, Brookhaven National Laboratory Technical Report No. BNL 51270, 1980.

[5] V. Ptitsyn, Ph.D. thesis, Budker Institute of Nuclear Physics, Novosibirsk, 1997 (in Russian).
[6] L. H. Thomas, Philos. Mag. 3, 1 (1927).

[7] V. Bargmann, L. Michel, and V.L. Telegdi, Phys. Rev. Lett. 2, 435 (1959).

[8] S. R. Mane, Nucl. Instrum. Methods Phys. Res., Sect. A 600, 383 (2009).

[9] Yu. M. Shatunov and S. R. Mane, Phys. Rev. ST Accel. Beams 12, 024001 (2009).

[10] M. Bai et al., Phys. Rev. E 56, 6002 (1997). 